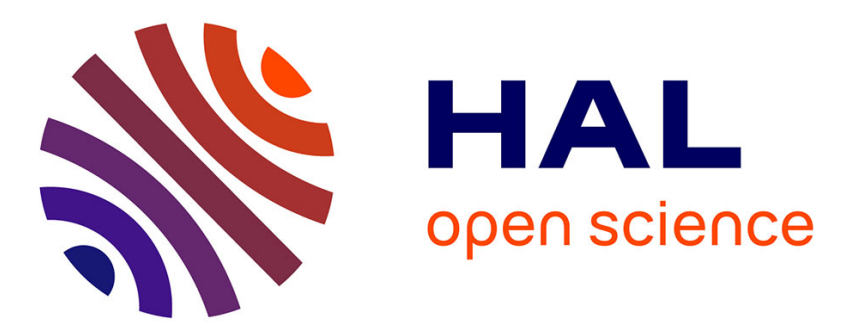

\title{
Pressure Transients in a Fractal-Cluster Model of Porous Media
}

\author{
Abraham de Swaan
}

\section{To cite this version:}

Abraham de Swaan. Pressure Transients in a Fractal-Cluster Model of Porous Media. Oil \& Gas Science and Technology - Revue d'IFP Energies nouvelles, 2016, 71 (1), pp.9. 10.2516/ogst/2014038 . hal-01707458

\section{HAL Id: hal-01707458 https://hal.science/hal-01707458}

Submitted on 12 Feb 2018

HAL is a multi-disciplinary open access archive for the deposit and dissemination of scientific research documents, whether they are published or not. The documents may come from teaching and research institutions in France or abroad, or from public or private research centers.
L'archive ouverte pluridisciplinaire HAL, est destinée au dépôt et à la diffusion de documents scientifiques de niveau recherche, publiés ou non, émanant des établissements d'enseignement et de recherche français ou étrangers, des laboratoires publics ou privés. 


\title{
Pressure Transients in a Fractal-Cluster Model of Porous Media
}

\author{
Abraham de Swaan* \\ Chilpa 16-4, Mexico D.F. cp 03910 - Mexico \\ e-mail: adeswaan@hotmail.com \\ * Corresponding author
}

\begin{abstract}
The reservoir is described as a "supercritical cluster"; that is, an aggregate of conductive elements that comprises a "backbone" of connected pores or fractures that span the zone of interest, and also a collection of "sub-critical clusters" or "dangling ends" joined to the backbone to a limited extent. The scheme resembles the usual fracture and matrix-blocks setting but both backbone and sub-clusters are of the same material and share similar petrophysical properties. Whereas the backbone is a homogeneous porous medium, the sub-critical clusters behave as fractal porous media. The backbone-cluster type of flow has been observed in laboratory experiments. The subcritical clusters were approximated as linear fractal media characterized by static and dynamic fractal exponents and also by porosity and permeability of the compound medium. One of the ends of the linear clusters is closed and the other is joined to the backbone, where the mainstream occurs. A new solution was developed for that problem. The Laplace transform in time and space was used in the mathematical scheme. The theory developed was applied to field cases of interference between wells in aquifers. The matches of computed and observed dynamic pressures show fair fits.
\end{abstract}

Résumé - Transitoires de pression dans un modèle d'agrégat fractal de milieux poreux Le réservoir est décrit comme un " agrégat supercritique »; à savoir un ensemble d'éléments conducteurs comprenant un "squelette » de pores connectés ou de fractures qui s'étendent sur la zone d'intérêt et également une collection " d'agrégats sous-critiques » ou " d'extrémités non saturées » jointes au squelette sur une distance limitée. Le schéma ressemble à la fracturation et aux assemblages blocs-matrice habituels mais le squelette et les sous-agrégats sont du même matériau et partagent des propriétés pétro-physiques similaires. Tandis que le squelette est un milieu poreux homogène, les agrégats sous-critiques se comportent comme des milieux poreux fractals. Ce type d'écoulement a été observé dans des expériences de laboratoire. Les agrégats sous-critiques ont été représentés comme des milieux fractals linéaires caractérisés par des exposants fractals statiques et dynamiques et également par la porosité et la perméabilité du milieu du composé. Une des extrémités des agrégats linéaires est fermée et l'autre est reliée au squelette, où coule le flux principal. Une nouvelle solution a été développée pour ce problème. La transformée de Laplace dans le temps et l'espace a été utilisée dans le développement mathématique. La théorie développée a été appliquée aux cas d'interférence sur le terrain entre les puits dans des aquifères. Les comparaisons de pressions dynamiques calculées et observées présentent des résultats compatibles. 


\section{NOMENCLATURE}

$f_{S C} \quad$ Fraction of SC-BB connected length

$A_{B B} \quad$ Well surface at the pay zone

$C \quad$ Coefficient in Equation (5)

d Fractal exponent

$h \quad$ Thickness (m)

$H \quad$ Heaviside step distribution

$\mathrm{K}_{v} \quad$ Modified Bessel function of order $v$

$L \quad$ Laplace transform

$L^{-1} \quad$ Inverse Laplace transform

$m \quad$ Integer

$p \quad$ Pressure $(\mathrm{Pa})$

$q \quad$ Flow rate $\left(\mathrm{m}^{3} / \mathrm{s}\right)$

$r \quad$ Radial distance $(\mathrm{m})$

$t \quad$ Time (s), (h in Fig. 9)

$x \quad$ Linear coordinate $(\mathrm{m})$

$\alpha \quad$ Fractal dimension exponent $\left(\equiv d_{f}\right)$

$\beta \quad$ Dynamic exponent $\left(=2 / d_{w}\right)$

$\theta \quad$ Exponent in Equation (2) in O'Shaugnessy and Procaccia (1985) and Barker (1988)

$\eta \quad$ Hydraulic diffusivity $\left(\mathrm{m}^{2} / \mathrm{s}\right)$

$\mu \quad$ Viscosity (Pa.s)

$\tau \quad$ Time-Laplace transform parameter

$\kappa \quad$ Space-Laplace transform parameter

$\nabla_{r}^{2} \quad$ Radial Laplacian operator

\section{SUBSCRIPTS AND SUPERSCRIPTS}

$\begin{array}{ll}B B & \text { Backbone } \\ f & \text { Fractal dimension } \\ S C & \text { Subcritical cluster } \\ w & \text { Brownian or random-walk } \\ \tilde{F} & \text { Time-Laplace transform of } F \\ \hat{F} & \text { Space-Laplace transform of } F \\ v & =1-d_{f} / d_{w}, \text { order of Bessel K function in } \\ & \text { (Metzler et al. 1994) }\end{array}$

\section{INTRODUCTION}

\section{Characterization of Fractal Porous Media}

The main characteristics of fractal media are two exponents that are defined in deterministic fractals. $d_{f}$ is the fractal dimension: the exponent that links a scalar factor applied to a regular fractal object and the number of elements that result from that scale-up. For instance in the Sierpinsky gasket when the scale is doubled and the central triangle is discarded the number of new elements is three, Figure 1.

In actual porous or fractured media $d_{f}$ is obtained from sampling and through box counting or sand box algorithms. See ben-Avraham and Havlin (2000) and Schroeder (1990). The extent of pore area or volume; or the number of fractures in the sampling space, increases as the $d_{f}$ power of the augmented search radius.

The dynamic exponent, $d_{w}$, is derived considering Brownian stochastic motion of a particle in the medium. It relates the scalar factor to the number of possible steps between two extreme nodes in a fractal net, Figure 2.

Computation of $d_{w}$ even in deterministic media is difficult, numerical enumerations are often used, but the Alexander and Orbach (1982) conjecture, the ratio of the exponents is a constant, $d_{f} / d_{w}=2 / 3$, allows determination of the dynamic from the fractal exponent.

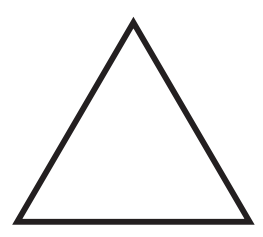

Figure 1

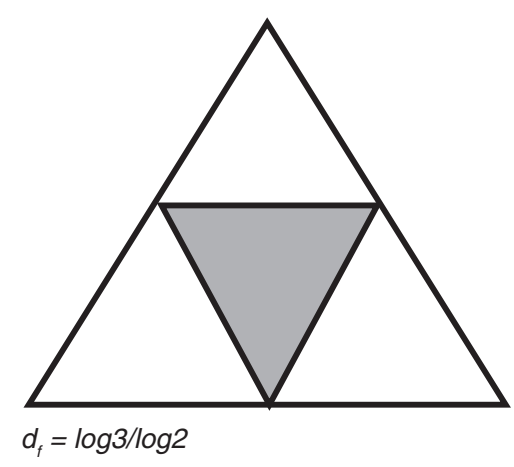

Upscaling in the Sierpinsky gasket.

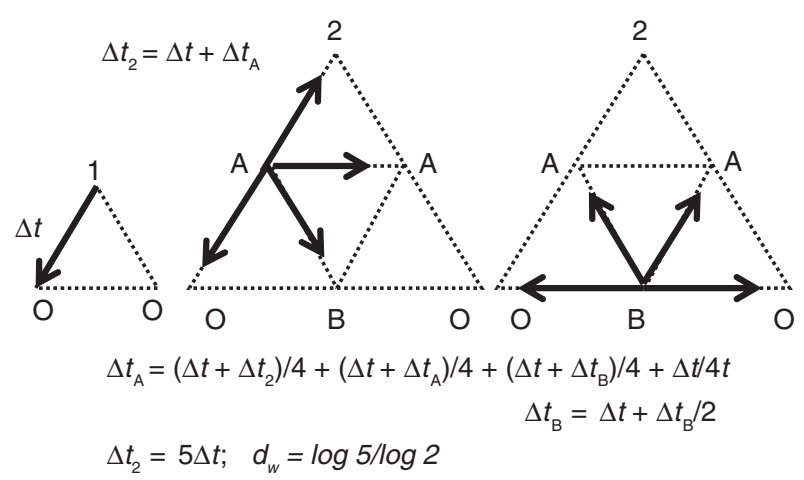

Figure 2

Brownian motion in the Sierpinsky gasket. 


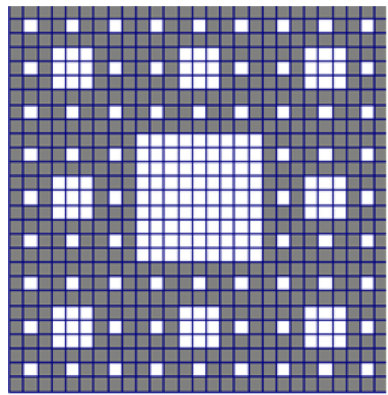

a)

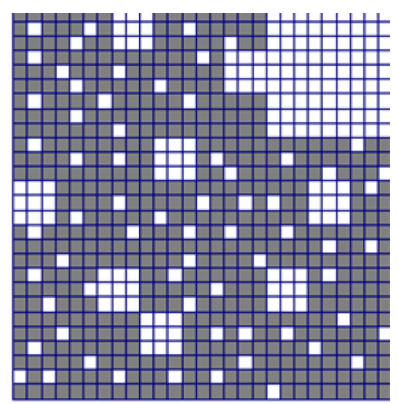

b)

Figure 3

a) Deterministic and b) random fractals.

That ratio has been proven as accurate by a number of experimental numerical studies (Rammal et al., 1984; Essam and Bhatti, 1985) although it is still a conjecture, i.e. with no analytical proof. In this paper, we adopt that link between static and dynamic exponents.

It is essential for the application of fractal principles to natural materials that random or stochastic fractals, representative of natural objects, preserve the properties of deterministic fractals, thus, the fractal in Figure $3 \mathrm{~b}$ may behave similarly to the one in Figure $3 \mathrm{a}$ in actual flow processes, (Ben-Avraham and Havlin, 2000).

Generally, the values of the exponents are fractions not as simple as the ratios shown in Figures 1 and 2.

The fractal diffusion equation:

$$
\nabla_{r}^{\alpha} p(r, t)-\eta \frac{\partial^{\beta} p(r, t)}{\partial t^{\beta}}=0
$$

with $\alpha \equiv d_{f}, \beta=2 / d_{w}$, has a foundation on probabilistic considerations with Fourier and Laplace transforms. The step from diffusion in fractal media to the fractional-derivative representation was not immediate but required the formulation of probabilistic concepts, (Compte, 1996; Gorenflo and Mainardi, 1997; Metzler and Klafter, 2000; Mainardi et al., 2001; Vlahos et al., 2008).

The methods for porous or for fractured media are the same; "Porous and fractured media appear to follow the same idealized scaling rule for both flow and transport" (Neuman, 1990). By using scalar arguments O'Shaugnessy and Procaccia (1985) and Barker (1988) transformed Equation (1) into a differential equation with a space dependent diffusivity:

$$
\eta \frac{\partial p}{\partial t}=\frac{1}{r^{d_{f}-1}} \frac{\partial}{\partial r}\left(r^{d_{f}-1-\theta} \frac{\partial p}{\partial r}\right)
$$

$\theta$ includes $d_{w}$. Basically it results from passing the scaling exponential law of distances to the geometrical material balance. Its solution is:

$$
p(r, t)=\frac{d_{w}}{d_{f} \Gamma\left(\frac{d_{f}}{d_{w}}\right)}\left(\frac{1}{a d_{w}{ }^{2}}\right)^{\frac{d_{f}}{d_{w}}} \exp \left(-\frac{r^{d_{w}}}{a d_{w}{ }^{2} t}\right)
$$

this and similar solutions have been labeled "stretched exponentials", (Sahimi, 1995; Metzler and Klafter, 2000). Chang and Yortsos (1990) applied it to the analysis of well tests. The solution, Equation (3), does not account for interference tests (Sahimi, 1993; Camacho Velazquez et al., 2008). It has been criticized for limiting diffusion as only space dependent but time independent (Sahimi, 1993; Leveinen, 2000).

A modification of Equation (2) was presented by Metzler et al. (1994) with a fractional time derivative dependent on $d_{w}$. (Raghavan, 2011).

$$
\eta \frac{\partial^{\beta} p}{\partial t^{\beta}}=\frac{1}{r^{2 d_{f} / d_{w}-1}} \frac{\partial}{\partial r}\left(r^{2 d_{f} / d_{w}-1} \frac{\partial p}{\partial r}\right)
$$

Its solution in the Laplace domain is:

$$
\tilde{p}(r, \tau)=C\left(\frac{r}{\tau^{\frac{2}{d_{w}}}}\right)^{1-\frac{d}{2}} K_{v}\left(r \tau^{d_{w}}\right)
$$

It has the advantage of describing interference, (de Swaan et al., 2012), and it does reduce to the normal case for integer exponents.

\section{Percolation Clusters}

The concept of "percolation cluster" as representative of porous and fractured media has been advanced by several authors. (Sahimi, 1995; ben Avraham and Havlin, 2000). They have been called "spatially periodic structures [...] homogeneous in the large scale but with a fractal microstructure" by Adler and Thovert (1993).

It has been reckoned that in fractured reservoirs "flow takes place in a relatively small number of fractures" (Leveinen, 2000) and "Only six fractures could be traced through nine sections [in a rock block with 240 fractures]", (Adler and Thovert, 1999, page 213). Figure 4.

A cluster of bounding elements becomes connected across a region of interest when the probability of an element of area being a conducting pore or bond, is greater than a fraction that depends on the underlying type of lattice, (see page 11 table 2.1 in Sahimi (1994); ben Avraham and Havlin, 2000). A cluster connected throughout is a "super-critical" cluster, Figure 5a. It is 


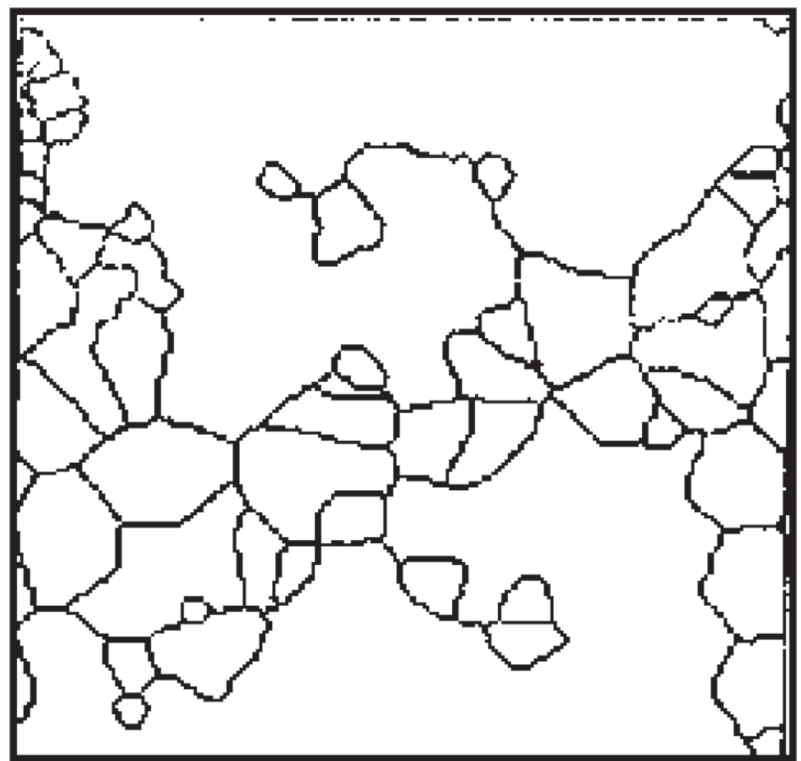

Figure 4

Fracture network, Adler and Thovert (1999).

composed by one or several BackBones (BB) communicated to Sub-critical Clusters (SC); that is, aggregates of pores or fractures with a connection to the backbone but isolated among them, Figure $5 \mathrm{~b}$.

The backbone is a homogeneous medium whereas the sub-critical clusters, as randomly generated aggregates, behave as fractal media. Thus, in the backbone flow of fluids follows the normal diffusion law in a connected medium. The backbone-cluster type of flow has been observed in laboratory experiments (Klemm et al., 1999). A turbiditic system (Chen et al., 2012) with communicating channels and appended lobes may also be approximated by the BB-SC description - in this case with different properties.

\section{THEORY}

\subsection{Diffusion in a Supercritical Cluster}

The system BB plus SC is similar to the well known system of fracture and matrix-rock blocks (de Swaan, 1986), but in this case both media share the same petrophysical properties: local porosity, permeability and rock compressibility.

BB's generally have varying cross sections along their lengths. They have "hot spots" where the section reduces to a single communicating element (fracture or pore); in between, they may have wide openings or "blobs". In the

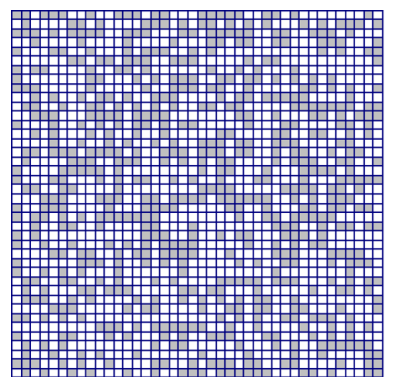

a)

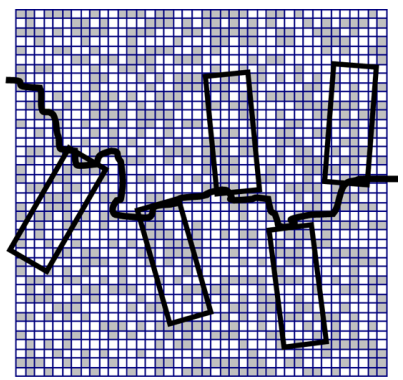

b)
Figure 5

Supercritical cluster a) and its description as backbone and subcritical clusters b).

present theory $\mathrm{BB}$ are assumed with a constant or homogeneous cross-section $h_{B B}$. Heterogeneous BB can be accounted for by composite linear, (Carslaw and Jaeger, 1959), radial or spherical regions, the last two with no variation in angular directions, (de Swaan, 1998).

We assumed that flow in the compound medium of backbones and fractal clusters is described by the following simplifying conditions:

1. The backbone is a uniform net of pores or fractures with average thickness $h_{B B}$;

2. Tortuosity of the backbone was not considered;

3. The SC are prisms with an average length $h_{S C}$ perpendicular to the backbone;

4. The SC contact a fraction $f_{S C}$ of the backbones' length, Figure 5;

5. Values of local porosity, permeability and compressibility are common to both media;

6. A repetitive element within the pay zone has a thickness equal to $h_{B B}+h_{S C}$.

The system for a single extended backbone is depicted in Figure 6.

In radial - axial - symmetry the pressure equation is:

$\nabla_{r}^{2} p_{B B}(r, t)-\eta \frac{\partial p_{B B}(r, t)}{\partial t}-\frac{f_{S C}}{h_{B B}} \int_{0}^{t} \frac{\partial p_{B B}\left(x, t^{\prime}\right)}{\partial t^{\prime}} q_{S C}\left(t^{\prime}\right) d t^{\prime}=0$

where $q_{S C}$ is the inflow from a representative or average $\mathrm{SC}$ to the $\mathrm{BB}$ as the effect of a unit step pressure disturbance at the contacting surface. The SC contact a fraction $f_{S C}$ along the radial BB's.

That outflow is convolved in time to account for the variable pressure in the backbone. The time-Laplace transform is:

$$
\nabla_{r}^{2} \tilde{p}_{B B}(r, t)-\eta \tau \tilde{p}_{B B}-\tau \frac{f_{S C}}{h_{B B}} \tilde{p}_{B B} \tilde{q}_{S C}(\tau)=0
$$




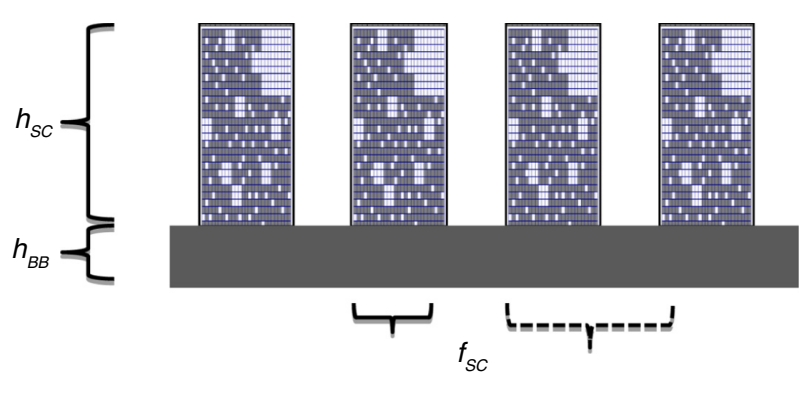

Figure 6

Schematic of backbone and subcritical clusters.

SC are considered with a linear finite geometry. One of the ends of the linear-fractal clusters is closed; the other extreme is connected to the BB. $\tilde{q}_{S C}$ is the transformed flow rate from a single SC after a unitary pressure disturbance - a heaviside step - in the BB.

The solution to Equation (7) in the time-Laplace domain is:

$$
\tilde{p}_{B B}=\frac{-q \mu}{A_{B B} k} \frac{K_{0}\left(r \sqrt{\tau / \eta^{\prime}}\right)}{\tau \sqrt{\tau / \eta^{\prime}} K_{1}\left(r_{w} \sqrt{\tau / \eta^{\prime}}\right)}
$$

where,

$$
\eta^{\prime}=\eta+\frac{f_{S C}}{h_{B B}} \tilde{q}_{S C}(\tau)
$$

Distributions of $h_{S C}$ lengths can be incorporated in the formulation as in the multiply sized blocks and fractures formulation, de Swaan (2000). Different BB and SC properties, as in turbiditic channel-lobe systems can also be represented in that scheme.

\subsection{Fractal Diffusion in a Finite Linear Medium}

The fractional differential equation:

$$
\frac{\partial^{\alpha} p}{\partial x^{\alpha}}=\eta \frac{\partial^{\beta} p}{\partial t^{\beta}}
$$

has been solved in several papers for infinite linear media, (Metzler and Klafter, 2004), but not for finite media with the boundary conditions of the present problem.

The clusters-to-backbone flow term in Equation (6) is accounted for through the solution to the fractional diffusion of pressure equation in a finite linear medium, $\alpha \equiv d_{f}, \beta=\frac{2}{d_{w}}$. Fractional derivative definitions include Riemann-Liouville, Grunwald-Letnikov, Weyl, Caputo, Marchaud, and Riesz, (Agrawal, 2002; Oldham and Spanier, 1974; Miller and Ross, 1993; Podlubny, 1999; Butzer and Westphal, 2000). Of the various derivatives we chose Caputo's which Laplace transform is:

$$
L_{x, \kappa}\left\{\frac{\partial^{\alpha} p}{\partial x^{\alpha}}\right\}=\kappa^{\alpha} \hat{p}-\sum_{j=0}^{m-1} \kappa^{\alpha-1-j} p^{(j)}(0+, t) ; m-1<\alpha \leq m
$$

where $m$ is an integer; $p^{(j)}$ is the derivative of order $j$. Thus, the $L$ transform of the Caputo time derivative is:

$$
L_{t, \tau}\left\{\frac{\partial^{\beta} p}{\partial t^{\beta}}\right\}=\tau^{\beta} \tilde{p}-\tau p(x, 0) ; \beta<2
$$

The Laplace transform of the Caputo derivative has the convenience of including integer derivatives, in contrast with Sturm-Liouville and other definitions of fractal derivatives.

The boundary condition at the extreme length $h_{S C}$ of the average sub-critical clusters is a Heaviside unit step function:

$$
p\left(h_{S C}, t\right)=H(t)
$$

with transform:

$$
\tilde{p}\left(h_{S C}, \tau\right)=1 / \tau
$$

As the space coordinate extends only in one direction from the origin, the Laplace transform can be used instead of the Fourier transformation. Circumflex means space-Laplace transform; a dash means the time-Laplace transform.

At $x=0$ the boundary condition is no-flow, we use the gradient as the regular order one derivative:

$$
\frac{\partial p(0, t)}{\partial x}=0
$$

or,

$$
\kappa \hat{p}(0, t)=0
$$

Space and time transforms of Equation (10) result in:

$$
\hat{\tilde{p}}(\kappa, \tau)=\frac{\kappa \tilde{p}(0, \tau)}{\kappa^{\alpha}-(\tau / \eta)^{\beta}}
$$




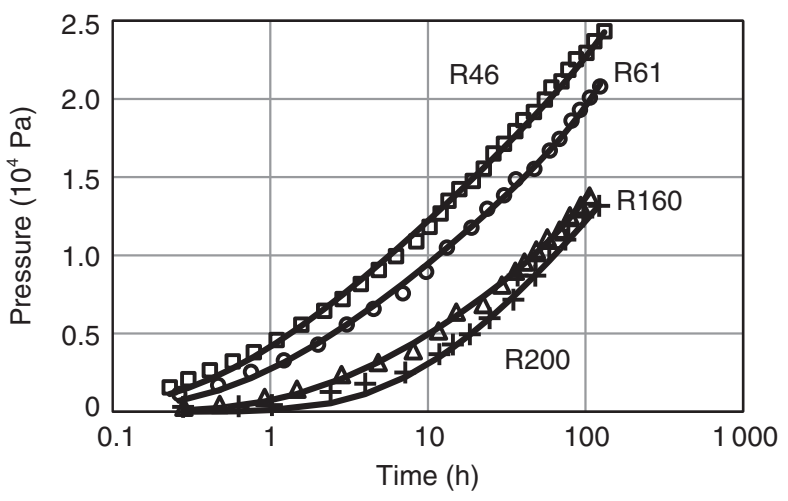

Figure 7

This theory (lines); observations (marks, Le Borgne et al., 2004).

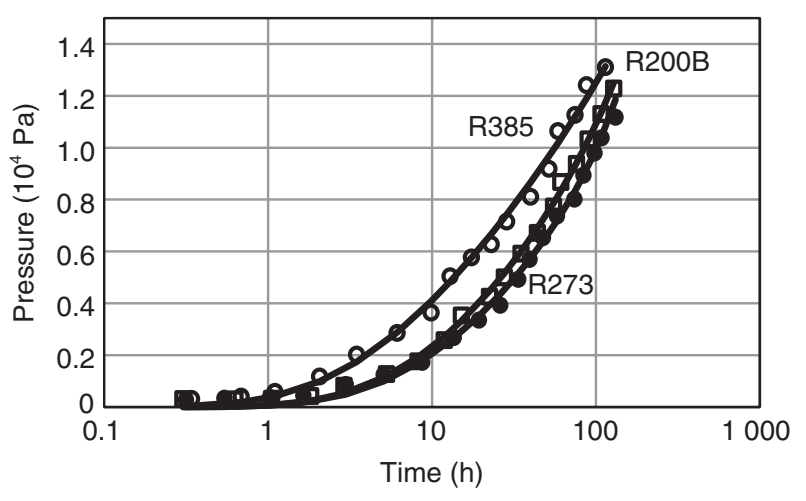

Figure 8

This theory (lines); observations (marks; Le Borgne et al., 2004).
Notice that Equation (16) is space-transformed and the numerator in Equation (17) is time-transformed. The boundary condition at $h_{S C}$, Equation (14), requires:

$$
\tilde{p}(0, \tau)=\frac{1}{\tau}\left[L_{\kappa, x}^{-1}\left\{\frac{\kappa}{\kappa^{\alpha}-(\tau / \eta)^{\beta}}\right\}\right]_{x=h_{S C}}^{-1}
$$

For $\alpha=2$ and $\beta=1$, the diffusivity equation reduces to the normal or Gaussian case as:

$$
L^{-1}\left\{\frac{\kappa}{\kappa^{2}-a^{2}}\right\}=\cosh (a x)
$$

and

$$
\tilde{p}(x, \tau)=\frac{\cosh (\sqrt{\tau / \eta} x)}{\tau \cosh \left(\sqrt{\tau / \eta} h_{S C}\right)}
$$

See Carslaw and Jaeger (1959) page 309.

The flow into or from the fractal SC cluster is the derivative of pressure at the face $x=h_{S C}$ we use the regular, non-fractal, space derivative:

$$
\begin{gathered}
\widetilde{q_{S C}}=\frac{k}{\mu} \frac{\partial \tilde{p}\left(h_{S C}, \tau\right)}{\partial x} \\
L_{x, \kappa} L_{t, \tau}\left\{\frac{\partial p(x, t)}{\partial x}\right\}=\frac{\kappa^{2} \tilde{p}(0, \tau)}{\tau\left[\kappa^{\alpha}-(\tau / \eta)^{\beta}\right]}
\end{gathered}
$$

The $L$ transform does not include fractional derivatives at $t=0$, neither at $x=0$, because we used the Caputo derivative.
The reduction to the normal case $(\alpha=2, \beta=1)$ results in a hyperbolic sine divided by the hyperbolic cosine at the constant pressure face, (Carslaw and Jaeger, 1959).

\section{RESULTS}

The transformed Equation (8) with source term Equation (21) is back-transformed in time by means of the Crump (1976) and Schapery (1961) procedures. Validation of the formulation was made by comparing its results with the analytical normal results with exponents $\alpha=2, \beta=1$, (not shown).

The properties $d_{f}, \varphi, k, h_{B B}, h_{S C}, f_{S C}$, were adjusted to match observed interference pressure tests by an automatic match procedure, (Minpack, 1999).

Figures 7 and 8 show results matched to punctual data selected from Le Borgne et al. (2004), from tests in a highly heterogeneous fractured aquifer. The names of the wells include their distance to the pumping well. The pressure increments at the observation wells show up at intervals unrelated to those distances. Tidal effects were not filtered.

Figure 9 shows results matched to observations from Bernard et al. (2006).

Table 1 contains values of the properties derived from the matches. Table 2 shows values used in the model.

Some adjusted values show lack of consistency; in wells R200 and M5, the SC and BB thicknesses add significantly to more than the corresponding aquifer thickness.

Most of the $d_{f}$ values in column 3 are between 1 and 2 . That value means that the trajectory of flow is a crooked line that covers more than the linear dimension, one; 
but less than the areal dimension (two), see the Koch line in Ben-Avraham and Havlin (2000) page 4. The exception is well M1 with a value of 2.69 which means a flow path close to be space-filling, (dimension 3).

By using a different technique, Bernard et al. (2006) give values of permeability of $4.5 \mathrm{E}-12 \mathrm{~m}^{2}$; porosities from 0.005 to 0.08 , from the pressure responses in the wells. Well M5 is in a perpendicular direction

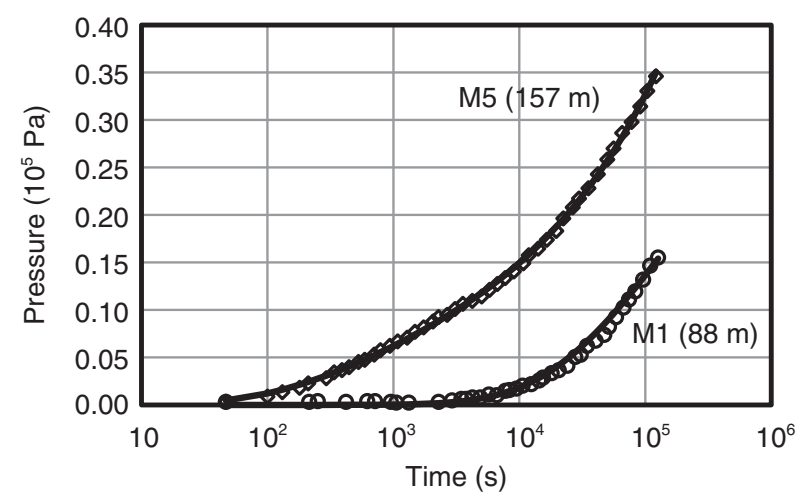

Figure 9

This theory (lines); observations (Bernard et al., 2006). from, and farther from the injection well than, well M1. It shows an earlier response; therefore manifesting high anisotropy in the formation.

\section{COMMENTS}

The presented matches are not unique; they depend on the starting values of the assumed properties; those presenting plausible values of the properties were selected and are shown in Figures 7 to 9 with the values in Table 1.

A full validation of the presented approach requires determination of fractal exponents and fracture space distribution besides the average petrophysical properties of the media where the well tests are conducted. Detailed numerical simulations of fractal media may be used to that avail.

A more realistic backbone with varying cross section can be represented as a linear medium with a collection of linear media (Carslaw and Jaeger 1959). In radial coordinates a collection of linked coronae can account for radial (not angular) variations in properties or thickness, (de Swaan, 1998).

Discrete distributions of varying properties and size of the dangling ends can be described in the manner of

TABLE 1

Adjusted properties, wells R46 to R385 from Le Borgne et al. (2004); wells M1 and M5 from Bernard et al. (2006)

\begin{tabular}{l|c|c|c|c|c|c|c|c}
\hline Well & Distance $(\mathrm{m})$ & $d_{f}$ & $d_{w}$ & $\varphi$ & $k\left(\mathrm{~m}^{2}\right)$ & $f_{S C}$ & $h_{S C}(\mathrm{~m})$ & $h_{B B}(\mathrm{~m})$ \\
\hline R46 & 46 & 1.92 & 2.88 & 0.0015 & $2.77 \mathrm{E}-13$ & 0.035 & 55.18 & 53.13 \\
\hline R61 & 61 & 1.89 & 2.84 & 0.0021 & $2.53 \mathrm{E}-13$ & 0.08 & 47.47 & 84.58 \\
\hline R160 & 160 & 1.01 & 1.51 & 0.092 & $7.50 \mathrm{E}-13$ & 0.30 & 1.83 & 28.20 \\
\hline R200 & 200 & 1.68 & 2.52 & 0.0078 & $2.76 \mathrm{E}-13$ & 0.11 & 36.64 & 100.9 \\
\hline R200B & 200 & 1.87 & 2.80 & 0.072 & $4.55 \mathrm{E}-13$ & 0.13 & 25.34 & 24.60 \\
\hline R273 & 273 & 1.83 & 2.75 & 0.044 & $3.53 \mathrm{E} 13$ & 0.42 & 27.53 & 67.07 \\
\hline R385 & 385 & 1.64 & 2.46 & 0.047 & $8.80 \mathrm{E}-13$ & 0.0049 & 11.70 & 23.55 \\
\hline M1 & 88 & 2.69 & 4.04 & 0.00069 & $1.07 \mathrm{E}-15$ & 0.68 & 10.94 & 3.40 \\
\hline M5 & 157 & 1.87 & 2.81 & 0.042 & $2.10 \mathrm{E}-13$ & 0.19 & 242.11 & 34.05 \\
\hline
\end{tabular}

TABLE 2

Aquifer interference tests and water properties

\begin{tabular}{l|c|c|c|c}
\hline Aquifer reference & Thickness & Water injection rate & Water compressibility & Water viscosity \\
\hline Le Borgne et al. (2004) & $100 \mathrm{~m}$ & $34 \mathrm{~m}^{3} / \mathrm{h}$ & $8.3 \mathrm{E}-10 \mathrm{~Pa}^{-1}$ & $0.17 \mathrm{E}-3 \mathrm{~Pa} . \mathrm{s}$ \\
\hline Bernard et al. (2006) & $120 \mathrm{~m}$ & $0.0187 \mathrm{~m}^{3} / \mathrm{s}$ & $8.3 \mathrm{E}-10 \mathrm{~Pa}^{-1}$ & $0.17 \mathrm{E}-3 \mathrm{~Pa} . \mathrm{s}$ \\
\hline
\end{tabular}


distributions of matrix-rock blocks in fractured reservoirs, (de Swaan, 2000).

A full characterization of actual porous or fractured reservoirs in terms of backbone(s) and dangling-end sizes, as well as indicators of fractal exponents is not known to the author. That knowledge is important for secondary recovery practices since in dangling ends fluids displacement is not operative; they are stagnant unproductive zones.

\section{CONCLUSIONS}

1. The diffusion equation with fractional space and time derivatives, representing flow in a finite, fractal and linear medium, was formulated by using the Caputo fractional derivative both in the space and time terms. The presented approach was validated with comparisons to the normal case with integer exponents;

2. The resulting outflow function from that linear medium was included as a source term in the equation of pressure transient in an infinite radial connected medium. That global equation was back-transformed - in time and space - with approximate procedures and matched to actual pressure-interference data from tests in aquifers, showing the feasibility of the approach;

3. The present theory provides a mathematical description of transient flow in media that may be represented as clusters of pores or fractures formed by backbone(s) and subcritical clusters.

\section{ACKNOWLEDGMENTS}

The author acknowledges valuable help from Rodolfo Camacho-Velazquez, Mario Vásquez-Cruz and Gabriela Gomez-Ortiz in the development of this paper. Figure 4, from Adler and Thovert (1999), is reproduced with kind permission from Springer Science + Business Media B.V.

Copyright 2013, Society of Petroleum Engineers, permission to publish 3307140398780 , Jan. 13th 2014. This paper was presented at the EAGE Annual Conference \& Exhibition Incorporating SPE Europec held in London, United Kingdom, 10-13 June 2013 as SPE Paper 164892.

\section{REFERENCES}

Adler P.M., Thovert J.F. (1993) Fractal Porous Media, Transport in Porous Media 13, 41-78.
Adler P.M., Thovert J.F. (1999) Fractures and Fracture Networks, Kluwer, Dordrecht.

Agrawal O.P. (2002) Solution for a Fractional Diffusion-Wave Equation Defined in a Bounded Domain, Nonlinear Dynamics 29, 145-155.

Alexander S., Orbach R. (1982) Density of states on fractals: 'fractons', J. Phys. Lett. 43, L625-631.

Barker J.A. (1988) A generalized radial flow model for hydraulic tests in fractured rock, Water Resour. Res. 24, 10, 1796-1804.

Ben-Avraham D., Havlin S. (2000) Diffusion and Reactions in Fractals and Disordered Systems, Cambridge, Massachusetts, Cambridge U Press.

Bernard S., Delay F., Porel G. (2006) A new method of data inversion for the identification of fractal characteristics and homogenization scale from hydraulic pumping tests in fractured aquifers, $J$ Hydrology 328, 647-658.

Butzer P.L., Westphal U. (2000) An introduction to fractional calculus, Hilfer R. (ed.), Applications of Fractional Calculus in Physics, World Scientific, Singapore, pp. 1-85.

Camacho Velazquez R., Fuentes-Cruz G., Vásquez-Cruz M. (2008) Decline Curve Analysis of Fractured Reservoirs with Fractal Geometry, SPE Reservoir Evaluation \& Engineering 11, 03, 606-619.

Carslaw H.S, Jaeger J.C. (1959) Conduction of Heat in Solids, 2nd ed., Oxford U. Press, New York.

Chang J., Yortsos Y.C. (1990) Pressure Transient Analysis of Fractal Reservoirs, SPE Formation Evaluation 5, 01, 31-38.

Chen T., Noirot J.-C., Khandelwal A., Xue Guangri, Barton M.D., Alpak F.O. (2012) Estimating Stratigraphic Parameters from Well Test Data in Turbidite Reservoirs, SPE Annual Technical Conference and Exhibition, 8-10 Oct, San Antonio TX, USA, SPE Paper 159090.

Compte A. (1996) Phys. Rev. E 53, 4, 4191-4193.

Crump K.S. (1976) Numerical Inversion of Laplace Transforms Using a Fourier Series Approximation, Journal ACM 23, 1, 89-96.

de Swaan A. (1986) Influence of Shape and Skin of MatrixRock Blocks on Pressure Transients in Fractured Reservoirs, 61st Annual Technical Conference and Exhibition of the Society of Petroleum Engineers, 5-8 Oct, New Orleans, LA, SPE Paper 15637.

de Swaan A. (1998) Transient Fluid Flow through Composite Geometries, SPE International Petroleum Conference and Exhibition of Mexico, 3-5 March, Villahermosa Mexico, SPE Paper 36777.

de Swaan A. (2000) Analysis of Well Tests in Multiple Fractured Reservoirs - Field Case Applications, SPE International Petroleum Conference and Exhibition, 1-3 Feb, Villahermosa, Mexico, SPE Paper 59015.

de Swaan A., Camacho-Velasquez R., Vasquez-Cruz M. (2012) Interference Tests Analysis in Fractured Formations with a Time-Fractional Equation, SPE Latin American and Caribbean Petroleum Engineering Conference, 16-18 April, Mexico-City, Mexico, SPE Paper 153615-PP.

Essam J.W., Bhatti F.M. (1985) Series expansion evidence supporting the Alexander-Orbach conjecture in two dimensions, J. Phys. A 18, 3577-3584. 
Gorenflo R., Mainardi F. (1997) Fractional calculus: integral and differential equations of fractional order, Carpinteri A., Mainardi F. (eds), Fractals and Fractional Calculus in Continuum Mechanic, Springer Verlag, Vienna, pp. 223-276.

Klemm A., Müller H.P., Kimmich R. (1999) Evaluation of fractal parameters of percolation model objects and natural porous media by means of NMR microscopy, Physica A: Statistical Mechanics and its Applications 266, 1-4, 242-246.

Le Borgne T., Bour O., de Dreuzy J.R., Davy P., Touchard F. (2004) Equivalent mean flow models for fractured aquifers: Insights from a pumping tests scaling interpretation, Water. Resour. Res. 40, W03512.

Leveinen J. (2000) Composite model with fractional flow dimensions for well test analysis in fractured rocks, J. Hydrol. 234, 116-141.

Mainardi F., Luchko Y., Pagnini G. (2001) Fractional Calculus and Applied Analysis 4, 2, 153-192.

Metzler R., Klafter J. (2000) The random walk's guide to anomalous diffusion: a fractional dynamics approach, Physics Reports 339, 1-77.

Metzler R., Klafter J. (2004) The restaurant the end of the random walk: recent developments in the description of anomalous transport by fractional dynamics, J. Phys. A: Math. Gen. 37, R161-R208.

Metzler R., Glockle W.G., Nonenmacher T.F. (1994) Fractional model equation for anomalous diffusion, Physica A 211, 13-24.

Miller K.S., Ross B. (1993) An Introduction to the Fractional Calculus and Fractional Differential Equations, Wiley, New York.

Minpack (1999) www.netlib.org.

Neuman S.P. (1990) Universal scaling of hydraulic conductivities and dispersivities in geologic media, Water Res. Research 26, 8, 1749-1758.
O’Shaugnessy B., Procaccia I. (1985) Analytical solutions for diffusion on fractal objects, Phys. Rev. Lett. 54, 5, 455-458.

Oldham K.B., Spanier J. (1974) The Fractional Calculus, Academic Press, New York.

Podlubny I. (1999) Fractional Differential Equations, Academic Press, New York.

Raghavan R. (2011) Fractional derivatives: Application to transient flow, Journal of Petroleum Science and Engineering 80, 1, 7-13.

Rammal R., Angles D'Auriac J.C., Benoit A. (1984) Universality of the spectral dimension of percolation clusters, Phys. Rev. B 30, 4087.

Sahimi M. (1993) Fractal and Superdiffusive Transport and Hydrodynamic Dispersion in Heterogeneous Poroous Media, Transport in porous Media 13, 3-40.

Sahimi M. (1994) Applications of Percolation Theory, Taylor and Francis, Bristol.

Sahimi M. (1995) Flow and Transport in Porous Media and Fractured Rock: From Classical Methods to Modern Approaches, VCH, Weinheim, pp. 204-205.

Schapery A. (1961) Approximate Methods of Transform Inversion for Viscoelastic Stress Analysis, Technical Report, 4th US National Congress Appl. Math.

Schroeder M. (1990) Fractals, Chaos, Power Laws, Freeman, New York.

Vlahos L., Isliker H., Kominis Y., Hizanidis K. (2008) Normal and Anomalous Diffusion: A Tutorial, arXiv:0805.0419v1.

Manuscript submitted in January 2014 Manuscript accepted in June 2014 Published online in November 2014 